Korean J. Math. 19 (2011), No. 1, pp. 77-85

\title{
QUADRATIC MAPPINGS ASSOCIATED WITH INNER PRODUCT SPACES
}

\author{
SUNG JIN LEE
}

Abstract. In [7], Th.M. Rassias proved that the norm defined over a real vector space $V$ is induced by an inner product if and only if for a fixed integer $n \geq 2$

$$
\sum_{i=1}^{n}\left\|x_{i}-\frac{1}{n} \sum_{j=1}^{n} x_{j}\right\|^{2}=\sum_{i=1}^{n}\left\|x_{i}\right\|^{2}-n\left\|\frac{1}{n} \sum_{i=1}^{n} x_{i}\right\|^{2}
$$

holds for all $x_{1}, \cdots, x_{n} \in V$.

Let $V, W$ be real vector spaces. It is shown that if an even mapping $f: V \rightarrow W$ satisfies

$$
\sum_{i=1}^{2 n} f\left(x_{i}-\frac{1}{2 n} \sum_{j=1}^{2 n} x_{j}\right)=\sum_{i=1}^{2 n} f\left(x_{i}\right)-2 n f\left(\frac{1}{2 n} \sum_{i=1}^{2 n} x_{i}\right)
$$

for all $x_{1}, \cdots, x_{2 n} \in V$, then the even mapping $f: V \rightarrow W$ is quadratic.

Furthermore, we prove the generalized Hyers-Ulam stability of the quadratic functional equation (0.1) in Banach spaces.

\section{Introduction}

The stability problem of functional equations was originated from a question of Ulam [15] concerning the stability of group homomorphisms. Hyers [5] gave a first affirmative partial answer to the question of Ulam for Banach spaces. Hyers' Theorem was generalized by Aoki [1] for additive mappings and by Th.M. Rassias [6] for linear mappings by considering an unbounded Cauchy difference. The paper of Th.M. Rassias

Received February 18, 2011. Revised March 8, 2011. Accepted March 10, 2011. 2000 Mathematics Subject Classification: 39B72, 46 C05.

Key words and phrases: quadratic mapping, quadratic functional equation, generalized Hyers-Ulam stability.

This work was supported by the Daejin University Research Grant in 2011. 
[6] has provided a lot of influence in the development of what we call generalized Hyers-Ulam stability of functional equations. A generalization of the Th.M. Rassias theorem was obtained by Găvruta [4] by replacing the unbounded Cauchy difference by a general control function in the spirit of Th.M. Rassias' approach.

A square norm on an inner product space satisfies the important parallelogram equality

$$
\|x+y\|^{2}+\|x-y\|^{2}=2\|x\|^{2}+2\|y\|^{2} .
$$

The functional equation

$$
f(x+y)+f(x-y)=2 f(x)+2 f(y)
$$

is called a quadratic functional equation. In particular, every solution of the quadratic functional equation is said to be a quadratic mapping. A generalized Hyers-Ulam stability problem for the quadratic functional equation was proved by Skof [14] for mappings $f: X \rightarrow Y$, where $X$ is a normed space and $Y$ is a Banach space. Cholewa [2] noticed that the theorem of Skof is still true if the relevant domain $X$ is replaced by an Abelian group. In [3], Czerwik proved the generalized HyersUlam stability of the quadratic functional equation. Several functional equations have been investigated in [8]-[13].

Throughout this paper, assume that $n$ is a fixed positive integer. Let $X$ be a real normed vector space with norm $\|\cdot\|$, and $Y$ a real Banach space with norm $\|\cdot\|$.

In this paper, we investigate the quadratic functional equation (0.1), and prove the generalized Hyers-Ulam stability of the quadratic functional equation (0.1) in Banach spaces.

\section{Quadratic mappings associated with inner product spaces}

We investigate the quadratic functional equation (0.1).

Lemma 2.1. Let $V$ and $W$ be real vector spaces. If an even mapping $f: V \rightarrow W$ satisfies

$$
\sum_{i=1}^{2 n} f\left(x_{i}-\frac{1}{2 n} \sum_{j=1}^{2 n} x_{j}\right)=\sum_{i=1}^{2 n} f\left(x_{i}\right)-2 n f\left(\frac{1}{2 n} \sum_{i=1}^{2 n} x_{i}\right)
$$

for all $x_{1}, \cdots, x_{2 n} \in V$, then the mapping $f: V \rightarrow W$ is quadratic, i.e.,

$$
f(x+y)+f(x-y)=2 f(x)+2 f(y)
$$


for all $x, y \in V$.

Proof. Assume that $f: V \rightarrow W$ satisfies (2.1).

Letting $x_{1}=\cdots=x_{n}=x, x_{n+1}=\cdots=x_{2 n}=y$ in (2.1), we get

$n f\left(x-\frac{x+y}{2}\right)+n f\left(y-\frac{x+y}{2}\right)=n f(x)+n f(y)-2 n f\left(\frac{x+y}{2}\right)$

for all $x, y \in V$. Since $f: V \rightarrow W$ is even,

$$
2 n f\left(\frac{x-y}{2}\right)=n f(x)+n f(y)-2 n f\left(\frac{x+y}{2}\right)
$$

for all $x, y \in V$. So

$$
2 f\left(\frac{x+y}{2}\right)+2 f\left(\frac{x-y}{2}\right)=f(x)+f(y)
$$

for all $x, y \in V$. Letting $x=y=0$ in (2.2), we get $f(0)=0$. Letting $y=0$ in (2.2), we get $f\left(\frac{x}{2}\right)=\frac{1}{4} f(x)$ for all $x \in V$. It follows from (2.2) that

$$
f(x+y)+f(x-y)=2 f(x)+2 f(y)
$$

for all $x, y \in V$.

Corollary 2.2. Let $V$ and $W$ be real vector spaces. An even mapping $f: V \rightarrow W$ satisfies

$$
\begin{aligned}
f\left(x-\frac{x+y}{2}\right) & +f\left(y-\frac{x+y}{2}\right) \\
& =f(x)+f(y)-2 f\left(\frac{x+y}{2}\right)
\end{aligned}
$$

for all $x, y \in V$ if and only if the mapping $f: V \rightarrow W$ is quadratic.

Proof. By Lemma 2.1, it is enough to show that if $f: V \rightarrow W$ is quadratic, then $f: V \rightarrow W$ satisfies (2.3).

Assume that $f: V \rightarrow W$ is quadratic. Since $f(2 x)=4 f(x)$ for all $x \in V, f\left(\frac{x}{2}\right)=\frac{1}{4} f(x)$ for all $x \in V$. So

$$
2 f\left(\frac{x+y}{2}\right)+2 f\left(\frac{x-y}{2}\right)=f(x)+f(y)
$$


for all $x, y \in V$. Thus

$$
\begin{aligned}
f\left(x-\frac{x+y}{2}\right) & +f\left(y-\frac{x+y}{2}\right) \\
& =2 f\left(\frac{x-y}{2}\right)=f(x)+f(y)-2 f\left(\frac{x+y}{2}\right)
\end{aligned}
$$

for all $x, y \in V$, as desired.

For a given mapping $f: X \rightarrow Y$, we define

$$
D f\left(x_{1}, \cdots, x_{2 n}\right):=\sum_{i=1}^{2 n} f\left(x_{i}-\frac{1}{2 n} \sum_{j=1}^{2 n} x_{j}\right)-\sum_{i=1}^{2 n} f\left(x_{i}\right)+2 n f\left(\frac{1}{2 n} \sum_{i=1}^{2 n} x_{i}\right)
$$

for all $x_{1}, \cdots, x_{2 n} \in X$.

Now we prove the generalized Hyers-Ulam stability of the quadratic functional equation $\operatorname{Df}\left(x_{1}, \cdots, x_{2 n}\right)=0$ in real Banach spaces.

Theorem 2.3. Let $f: X \rightarrow Y$ be a mapping satisfying $f(0)=0$ for which there exists a function $\varphi: X^{2 n} \rightarrow[0, \infty)$ such that

$$
\begin{aligned}
\widetilde{\varphi}\left(x_{1}, \cdots, x_{2 n}\right): & =\sum_{j=0}^{\infty} 4^{j} \varphi\left(\frac{x_{1}}{2^{j}}, \cdots, \frac{x_{2 n}}{2^{j}}\right)<\infty, \\
\left\|D f\left(x_{1}, \cdots, x_{2 n}\right)\right\| & \leq \varphi\left(x_{1}, \cdots, x_{2 n}\right)
\end{aligned}
$$

for all $x_{1}, \cdots, x_{2 n} \in X$. Then there exists a unique quadratic mapping $Q: X \rightarrow Y$ satisfying (2.1) such that

$$
\begin{aligned}
\| f(x)+ & f(-x)-Q(x) \| \\
& \leq \frac{1}{n} \widetilde{\varphi}(\underbrace{x, \cdots, x}_{n \text { times }}, \underbrace{0, \cdots, 0}_{n \text { times }})+\frac{1}{n} \widetilde{\varphi}(\underbrace{-x, \cdots,-x}_{n \text { times }}, \underbrace{0, \cdots, 0}_{n \text { times }})
\end{aligned}
$$

for all $x \in X$.

Proof. Letting $x_{1}=\cdots=x_{n}=x$ and $x_{n+1}=\cdots=x_{2 n}=0$ in (2.5), we get

$$
\begin{aligned}
\| 3 n f\left(\frac{x}{2}\right)+ & n f\left(\frac{-x}{2}\right)-n f(x) \| \\
& \leq \varphi(\underbrace{x, \cdots, x}_{n \text { times }}, \underbrace{0, \cdots, 0}_{n \text { times }})
\end{aligned}
$$


for all $x \in X$. Replacing $x$ by $-x$ in (2.7), we get

$$
\begin{aligned}
\| 3 n f\left(\frac{-x}{2}\right) & +n f\left(\frac{x}{2}\right)-n f(-x) \| \\
& \leq \varphi(\underbrace{-x, \cdots,-x}_{n \text { times }}, \underbrace{0, \cdots, 0}_{n \text { times }})
\end{aligned}
$$

for all $x \in X$. Let $g(x):=f(x)+f(-x)$ for all $x \in X$. It follows from (2.7) and (2.8) that

$$
\begin{aligned}
\| 4 n g\left(\frac{x}{2}\right) & -n g(x) \| \\
\leq & \varphi(\underbrace{x, \cdots, x}_{n \text { times }}, \underbrace{0, \cdots, 0}_{n \text { times }})+\varphi(\underbrace{-x, \cdots,-x}_{n \text { times }}, \underbrace{0, \cdots, 0}_{n \text { times }})
\end{aligned}
$$

for all $x \in X$. So

$$
\begin{aligned}
\| g(x)- & 4 g\left(\frac{x}{2}\right) \| \\
& \leq \frac{1}{n} \varphi(\underbrace{x, \cdots, x}_{n \text { times }}, \underbrace{0, \cdots, 0}_{n \text { times }})+\frac{1}{n} \varphi(\underbrace{-x, \cdots,-x}_{n \text { times }}, \underbrace{0, \cdots, 0}_{n \text { times }})
\end{aligned}
$$

for all $x \in X$. Hence

$$
\begin{aligned}
\left\|4^{l} g\left(\frac{x}{2^{l}}\right)-4^{m} g\left(\frac{x}{2^{m}}\right)\right\| & \leq \sum_{j=l}^{m-1} \frac{4^{j}}{n} \varphi(\underbrace{\frac{x}{2^{j}}, \cdots, \frac{x}{2^{j}}}_{n \text { times }}, \underbrace{0, \cdots, 0}_{n \text { times }}) \\
& +\sum_{j=l}^{m-1} \frac{4^{j}}{n} \varphi(\underbrace{-\frac{x}{2^{j}}, \cdots,-\frac{x}{2^{j}}}_{n \text { times }}, \underbrace{0, \cdots, 0}_{n \text { times }})
\end{aligned}
$$

for all nonnegative integers $m$ and $l$ with $m>l$ and all $x \in X$. It follows from (2.4) and (2.10) that the sequence $\left\{4^{k} g\left(\frac{x}{2^{k}}\right)\right\}$ is Cauchy for all $x \in X$. Since $Y$ is complete, the sequence $\left\{4^{k} g\left(\frac{x}{2^{k}}\right)\right\}$ converges. So one can define the mapping $Q: X \rightarrow Y$ by

$$
Q(x):=\lim _{k \rightarrow \infty} 4^{k} g\left(\frac{x}{2^{k}}\right)
$$

for all $x \in X$. 
By (2.4) and (2.5),

$$
\begin{aligned}
& \left\|D Q\left(x_{1}, \cdots, x_{2 n}\right)\right\|=\lim _{k \rightarrow \infty} 4^{k}\left\|D g\left(\frac{x_{1}}{2^{k}}, \cdots, \frac{x_{2 n}}{2^{k}}\right)\right\| \\
& \quad \leq \lim _{k \rightarrow \infty} 4^{k}\left(\varphi\left(\frac{x_{1}}{2^{k}}, \cdots, \frac{x_{2 n}}{2^{k}}\right)+\varphi\left(-\frac{x_{1}}{2^{k}}, \cdots,-\frac{x_{2 n}}{2^{k}}\right)\right)=0
\end{aligned}
$$

for all $x_{1}, \cdots, x_{2 n} \in X$. So $D Q\left(x_{1}, \cdots, x_{2 n}\right)=0$. By Lemma 2.1, the mapping $Q: X \rightarrow Y$ is quadratic. Moreover, letting $l=0$ and passing the limit $m \rightarrow \infty$ in (2.10), we get (2.6). So there exists a quadratic mapping $Q: X \rightarrow Y$ satisfying (2.1) and (2.6).

Now, let $Q^{\prime}: X \rightarrow Y$ be another quadratic mapping satisfying (2.1) and (2.6). Then we have

$$
\begin{aligned}
& \left\|Q(x)-Q^{\prime}(x)\right\|=4^{q}\left\|Q\left(\frac{x}{2^{q}}\right)-Q^{\prime}\left(\frac{x}{2^{q}}\right)\right\| \\
& \begin{aligned}
\leq 4^{q}\left\|Q\left(\frac{x}{2^{q}}\right)-f\left(\frac{x}{2^{q}}\right)-f\left(\frac{-x}{2^{q}}\right)\right\| \\
\quad+4^{q}\left\|Q^{\prime}\left(\frac{x}{2^{q}}\right)-f\left(\frac{x}{2^{q}}\right)-f\left(\frac{-x}{2^{q}}\right)\right\| \\
\leq \frac{2 \cdot 4^{q}}{n} \widetilde{\varphi}(\underbrace{\frac{x}{2^{q}}, \cdots, \frac{x}{2^{q}}}_{n \text { times }}, \underbrace{0, \cdots, 0}_{n \text { times }})+\frac{2 \cdot 4^{q}}{n} \widetilde{\varphi}(\underbrace{\frac{-x}{2^{q}}, \cdots, \frac{-x}{2^{q}}}_{n \text { times }}, \underbrace{0, \cdots, 0}_{n \text { times }},),
\end{aligned}
\end{aligned}
$$

which tends to zero as $q \rightarrow \infty$ for all $x \in X$. So we can conclude that $Q(x)=Q^{\prime}(x)$ for all $x \in X$. This proves the uniqueness of $Q$.

Corollary 2.4. Let $p>2$ and $\theta$ be positive real numbers, and let $f: X \rightarrow Y$ be a mapping such that

$$
\left\|D f\left(x_{1}, \cdots, x_{2 n}\right)\right\| \leq \theta \sum_{j=1}^{2 n}\left\|x_{j}\right\|^{p}
$$

for all $x_{1}, \cdots, x_{2 n} \in X$. Then there exists a unique quadratic mapping $Q: X \rightarrow Y$ satisfying (2.1) such that

$$
\|f(x)+f(-x)-Q(x)\| \leq \frac{2^{p+1} \theta}{2^{p}-4}\|x\|^{p}
$$

for all $x \in X$.

Proof. Define $\varphi\left(x_{1}, \cdots, x_{2 n}\right)=\theta \sum_{j=1}^{2 n}\left\|x_{j}\right\|^{p}$, and apply Theorem 2.3 to get the desired result. 
Corollary 2.5. Let $f: X \rightarrow Y$ be an even mapping satisfying $f(0)=0$ for which there exists a function $\varphi: X^{2 n} \rightarrow[0, \infty)$ satisfying (2.4) and (2.5). Then there exists a unique quadratic mapping $Q: X \rightarrow$ $Y$ satisfying (2.1) such that

$$
\|f(x)-Q(x)\| \leq \frac{1}{n} \widetilde{\varphi}(\underbrace{x, \cdots, x}_{n \text { times }}, \underbrace{0, \cdots, 0}_{n \text { times }})
$$

for all $x \in X$, where $\widetilde{\varphi}$ is defined in (2.4).

THEOREM 2.6. Let $f: X \rightarrow Y$ be a mapping satisfying $f(0)=0$ for which there exists a function $\varphi: X^{2 n} \rightarrow[0, \infty)$ satisfying $(2.5)$ such that

$$
\widetilde{\varphi}\left(x_{1}, \cdots, x_{2 n}\right):=\sum_{j=1}^{\infty} 4^{-j} \varphi\left(2^{j} x_{1}, \cdots, 2^{j} x_{2 n}\right)<\infty
$$

for all $x_{1}, \cdots, x_{2 n} \in X$. Then there exists a unique quadratic mapping $Q: X \rightarrow Y$ satisfying (2.1) such that

$$
\begin{aligned}
\| f(x) & +f(-x)-Q(x) \| \\
\leq & \frac{1}{n} \widetilde{\varphi}(\underbrace{x, \cdots, x}_{n \text { times }}, \underbrace{0, \cdots, 0}_{n \text { times }})+\frac{1}{n} \widetilde{\varphi}(\underbrace{-x, \cdots,-x}_{n \text { times }}, \underbrace{0, \cdots, 0}_{n \text { times }})
\end{aligned}
$$

for all $x \in X$.

Proof. It follows from (2.9) that

$$
\begin{aligned}
\left\|g(x)-\frac{1}{4} g(2 x)\right\| \leq \frac{1}{4 n} \varphi(\underbrace{2 x, \cdots, 2 x}_{n \text { times }}, \underbrace{0, \cdots, 0}_{n \text { times }}) \\
+\frac{1}{4 n} \varphi(\underbrace{-2 x, \cdots,-2 x}_{n \text { times }}, \underbrace{0, \cdots, 0}_{n \text { times }})
\end{aligned}
$$

for all $x \in X$. So

$$
\begin{aligned}
\left\|\frac{1}{4^{l}} g\left(2^{l} x\right)-\frac{1}{4^{m}} g\left(2^{m} x\right)\right\| & \leq \sum_{j=l+1}^{m} \frac{1}{4^{j} n} \varphi(\underbrace{2^{j} x, \cdots, 2^{j} x}_{n \text { times }}, \underbrace{0, \cdots, 0}_{n \text { times }}) \\
& +\sum_{j=l+1}^{m} \frac{1}{4^{j} n} \varphi(\underbrace{-2^{j} x, \cdots,-2^{j} x}_{n \text { times }}, \underbrace{0, \cdots, 0}_{n \text { times }})
\end{aligned}
$$

for all nonnegative integers $m$ and $l$ with $m>l$ and all $x \in X$. It follows from (2.12) and (2.14) that the sequence $\left\{\frac{1}{4^{k}} g\left(2^{k} x\right)\right\}$ is Cauchy for all 
$x \in X$. Since $Y$ is complete, the sequence $\left\{\frac{1}{4^{k}} g\left(2^{k} x\right)\right\}$ converges. So one can define the mapping $Q: X \rightarrow Y$ by

$$
Q(x):=\lim _{k \rightarrow \infty} \frac{1}{4^{k}} g\left(2^{k} x\right)
$$

for all $x \in X$.

By (2.5) and (2.12),

$$
\begin{aligned}
& \left\|D Q\left(x_{1}, \cdots, x_{2 n}\right)\right\|=\lim _{k \rightarrow \infty} \frac{1}{4^{k}}\left\|D g\left(2^{k} x_{1}, \cdots, 2^{k} x_{2 n}\right)\right\| \\
& \quad \leq \lim _{k \rightarrow \infty} \frac{1}{4^{k}}\left(\varphi\left(2^{k} x_{1}, \cdots, 2^{k} x_{2 n}\right)+\varphi\left(-2^{k} x_{1}, \cdots,-2^{k} x_{2 n}\right)\right)=0
\end{aligned}
$$

for all $x_{1}, \cdots, x_{2 n} \in X$. So $D Q\left(x_{1}, \cdots, x_{2 n}\right)=0$. By Lemma 2.1, the mapping $Q: X \rightarrow Y$ is quadratic. Moreover, letting $l=0$ and passing the limit $m \rightarrow \infty$ in (2.14), we get (2.13). So there exists a quadratic mapping $Q: X \rightarrow Y$ satisfying (2.1) and (2.13).

The rest of the proof is similar to the proof of Theorem 2.3.

Corollary 2.7. Let $p<2$ and $\theta$ be positive real numbers, and let $f: X \rightarrow Y$ be a mapping satisfying (2.11). Then there exists a unique quadratic mapping $Q: X \rightarrow Y$ satisfying (2.1) such that

$$
\|f(x)+f(-x)-Q(x)\| \leq \frac{2^{p+1} \theta}{4-2^{p}}\|x\|^{p}
$$

for all $x \in X$.

Proof. Define $\varphi\left(x_{1}, \cdots, x_{2 n}\right)=\theta \sum_{j=1}^{2 n}\left\|x_{j}\right\|^{p}$, and apply Theorem 2.6 to get the desired result.

Corollary 2.8. Let $f: X \rightarrow Y$ be an even mapping satisfying $f(0)=0$ for which there exists a function $\varphi: X^{2 n} \rightarrow[0, \infty)$ satisfying (2.5) and (2.12). Then there exists a unique quadratic mapping $Q$ : $X \rightarrow Y$ satisfying (2.1) such that

$$
\|f(x)-Q(x)\| \leq \frac{1}{n} \widetilde{\varphi}(\underbrace{x, \cdots, x}_{n \text { times }}, \underbrace{0, \cdots, 0}_{n \text { times }})
$$

for all $x \in X$, where $\widetilde{\varphi}$ is defined in (2.12). 


\section{References}

[1] T. Aoki, On the stability of the linear transformation in Banach spaces, J. Math. Soc. Japan 2 (1950), 64-66.

[2] P.W. Cholewa, Remarks on the stability of functional equations, Aequationes Math. 27 (1984), 76-86.

[3] S. Czerwik, On the stability of the quadratic mapping in normed spaces, Abh. Math. Sem. Univ. Hamburg 62 (1992), 59-64.

[4] P. Găvruta, A generalization of the Hyers-Ulam-Rassias stability of approximately additive mappings, J. Math. Anal. Appl. 184 (1994), 431-436.

[5] D.H. Hyers, On the stability of the linear functional equation, Proc. Natl. Acad. Sci. USA 27 (1941), 222-224.

[6] Th.M. Rassias, On the stability of the linear mapping in Banach spaces, Proc. Amer. Math. Soc. 72 (1978), 297-300.

[7] Th.M. Rassias, New characterizations of inner product spaces, Bull. Sci. Math. 108 (1984), 95-99.

[8] Th.M. Rassias, On the stability of the quadratic functional equation and its applications, Studia Univ. Babes-Bolyai XLIII (1998), 89-124.

[9] Th.M. Rassias, The problem of S.M. Ulam for approximately multiplicative mappings, J. Math. Anal. Appl. 246 (2000), 352-378.

[10] Th.M. Rassias, On the stability of functional equations in Banach spaces, J. Math. Anal. Appl. 251 (2000), 264-284.

[11] Th.M. Rassias, On the stability of functional equations and a problem of Ulam, Acta Appl. Math. 62 (2000), 23-130.

[12] Th.M. Rassias and P. Šemrl, On the Hyers-Ulam stability of linear mappings, J. Math. Anal. Appl. 173 (1993), 325-338.

[13] Th.M. Rassias and K. Shibata, Variational problem of some quadratic functionals in complex analysis, J. Math. Anal. Appl. 228 (1998), 234-253.

[14] F. Skof, Proprietà locali e approssimazione di operatori, Rend. Sem. Mat. Fis. Milano 53 (1983), 113-129.

[15] S.M. Ulam, Problems in Modern Mathematics, Wiley, New York, 1960.

Department of Mathematics

Daejin University

Kyeonggi 487-711, Republic of Korea

E-mail: hyper@daejin.ac.kr 